\title{
A Case for Joint Development of IR Cameras in India
}

\author{
J. Govindarajan \\ Assistant Professor, IR Thermography Division, Institute for Plasma Research \\ Bhat, Gandhinagar, Gujarat - 382428 India \\ E-mail : govindarajan@ipr.res.in
}

\begin{abstract}
The paper will start by introducing Infrared Imaging and go on to highlight some important applications of these imagers in various fields. This will be followed by the portrayal of typical Infrared cameras. Then, a specific case of an uncooled microbolometer imager will be described. It will go on to emphasize what constituent technologies that are already available in India and what more need to be done in manufacturing these cameras locally. The paper will conclude with an appeal for joint development of these cameras with in India, by proper coordination between research and educational institutions and relevant industries.
\end{abstract}

Key words : Infrared cameras, Image Processing, Microbolometer cameras

\section{INTRODUCTION}

Infrared waves are part of the electromagnetic spectrum that lies between visible and microwave range $(0.9 \mathrm{~m}$ to $1000 \mathrm{~m}$ ). All bodies above 0 oK emit electromagnetic radiation, as given by Plank's radiation law.

$I(v, T)=\frac{2 \pi v^{3}}{c^{2}} \frac{1}{e^{\left(\frac{h v}{k T}\right)}-1}$

Where, $T$ is the temperature and the frequency of the radiation. Bodies at few to few thousand oK mainly emit in the infrared region. Wein's Dispalcement law that gives the occurrence of peak wavelength of emission is given as Fig. 1.

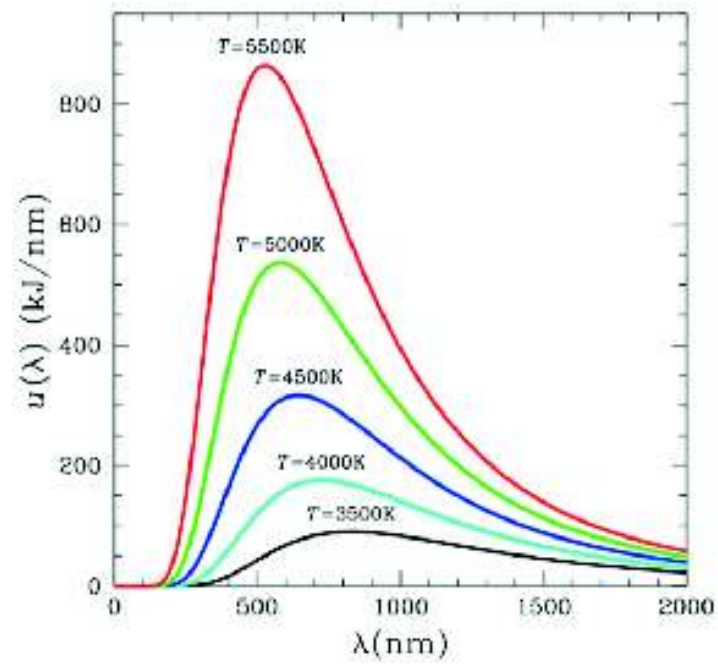

Fig. 1. Blackbody radiation and wein's displacementlaw [1] $\lambda_{\text {max }}=\frac{2.9 \times 10^{-3}}{T}$

Besides, the famous Stefan Botzmann law, gives the total emissive power of an ideal black body as a function of temperature is given by

$\frac{P}{A}=k T^{4}$

Where, $P$ is the power, $A$ the area of the emitting surface and $k$ the Stefan Botzmann constant.

Hence, in principle, this radiation helps in imaging the emitting object in Infrared (IR) region. However, one main thing to note is that unlike visible imaging that mainly depends upon the reflection of light from objects infrared imaging is done by the emitted radiation from the body itself. Hence, an external source is not necessary for infrared or thermal imaging.

Real bodies are not ideal absorbers or emitters as black bodies as given in Eq.1. A surface parameter called emissivity, whose value ranges in the interval 0 to 1 , determines the amount of radiation emitted as compared to ideal black body Fig. 2 .

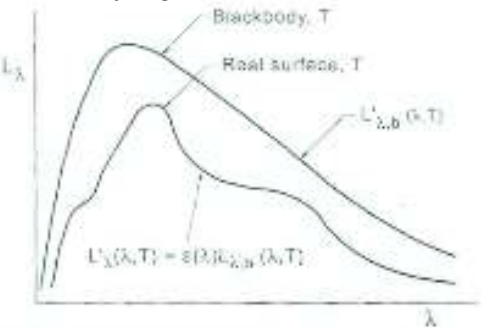

Fig.2. Real bodies and emissivity 
Moreover, the image transmission also is determined by the intervening media's absorption properties. It is important to note that due to many absorbing gases in the atmosphere infrared transmission occurs only in few windows of wavelengths, as given in Fig. 3 .

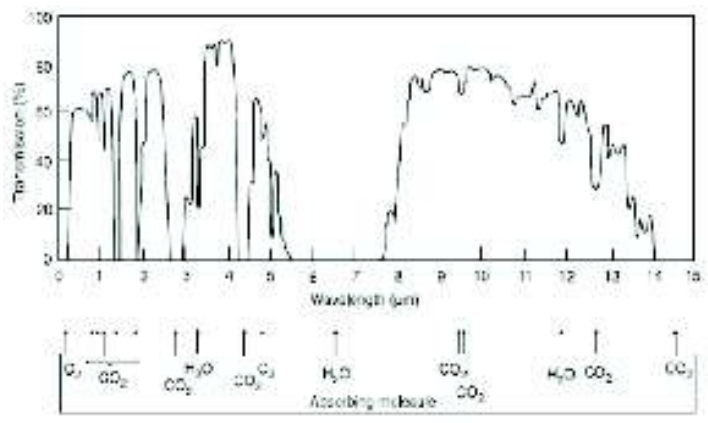

Fig.3. Atmospheric transmission

This has lead to dividing the whole infrared spectrum roughly in to four sub regions, as given in Table 1.

Table. 1. Infrared ranges

\begin{tabular}{|c|c|c|}
\hline NIR & $0.75 \quad 1.40$ & $\mu \mathrm{m}$ \\
\hline SWIR & 1.40 & $\mu \mathrm{m}$ \\
\hline MWIR & $3.00-8.00$ & $\mu \mathrm{m}$ \\
\hline I.WIIR & $8.00-15.00$ & $\mu \mathrm{m}$ \\
\hline FIR & $15.00 \quad 1000$ & $\mu \mathrm{m}$ \\
\hline
\end{tabular}

Hence, this has also lead to the commercial development of IR detectors and cameras appropriate to these four regions. The choice of the type of camera to be used depends upon the temperature range of the objects to be studied.

In the last few decades the applications of the thermal imaging infrared cameras have grown in leaps and bounds. This is mainly due to the developments in the fields of semiconductors, computers, sensor technology, Image processing etc. Presently theses imagers are being extensively used in areas as wide as manufacturing, non-destructive evaluation, process monitoring and routine preventive maintenance in industries. Civil engineers have started routinely using IR imagers for inspection of building for seepage studies and energy loss studies. Many automobile industries have started using them for improving driver's safety while driving during nights and fog. Moreover, IR cameras are also used by security agencies; police etc. due to their night vision capabilities. Besides, they have also found important applications in medical and veterinary fields. These cameras are also used in art and architectural restoration.

In India these cameras are so far generally imported.
Owing to the duel use nature of infrared imagers it is at times very difficult to procure them for even normal applications. Hence, there is an urgent need for developing the requisite skills and for manufacturing these imagers in India itself. The rest of the paper will deal with describing the constituent parts of these imagers, the technologies and skills that are required for fabricating them as well as the competence that are already existing with in India and finally what more needs to be done to achieve the goal of indigenous manufacture of these cameras.

\section{INFRARED IMAGERS}

The main constituents of an infrared camera, as shown in Fig. 4. are

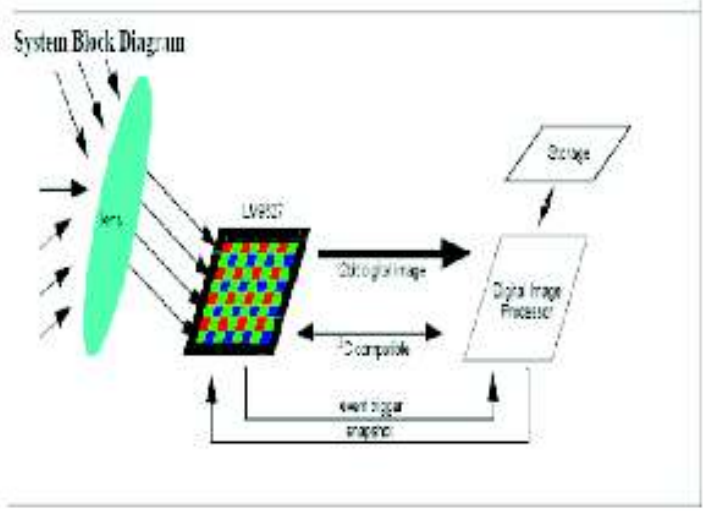

Fig. 4. Constituents of a digital camera

1. The imaging optics

2. The infrared sensors

3. The proximity electronics

4. Cryogenics and thermal stabilization

5. The data interface and camera control

6. Image processing and control software

\section{Imaging optics}

This optics is for the formation of the IR image on the image sensor. Like any other camera these may be made of multiple lenses for clear and aberration free imaging. The base material of these lenses depends upon the operational wavelength range of the camera to be fabricated.

\section{Infrared sensors}

IR detectors are devices that convert radiant IR energy into a measurable electrical signal like voltage or current. They are made of materials that are sensitive to 1-3 m, 3-5 $m$ or 8-12 $m$ wavelength ranges. There are mainly two 
types of detectors, Thermal and Photonic. Photonic detectors are sensors were the signal is obtained by direct measurement of electron and hole excitations generated by incident photons. In Thermal detectors, on the other hand, the incident IR radiation heats the surface, which affects some measurable property of the heated material. That is detected as the signal. The examples of the former are $\mathrm{HgCdTe}, \mathrm{InSb}$ or $\mathrm{PbS}$ detectors where as the later is exemplified by Pyrometers, Bolometers etc.

In the beginning single or linear array of detectors were used in the cameras and the image was obtained by scanning the scene by some mechanism of moving mirrors, as shown in Fig. 5.

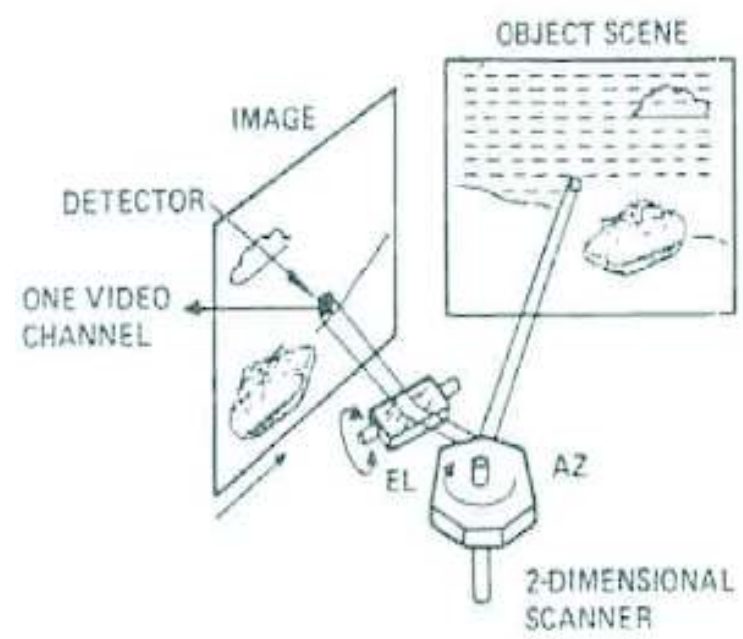

Fig. 5. Mechanically scanned IR camera [2]

However, the recent trend is to use a two dimensional array of detectors on the focal plane of the optics. These are called Focal Plane Array (FPA) sensors or Starring Array Sensors The main advantage in these type of sensors is that there is no mechanical motion and hence in principle very high frame rates cam be achieved. There are indeed two types of FPA, sensors with either CCD or CMOS based on the read out electronics. Infrared FPAs are mostly CMOS based and hence, in principle each element of the array, that is each pixel, can be addressed. It is worth noting that in the CMOS type detectors besides the IR sensitive area there is also an amplifier to improve the signal gain. The functioning of a CMOS type detector is shown in Fig. 6.

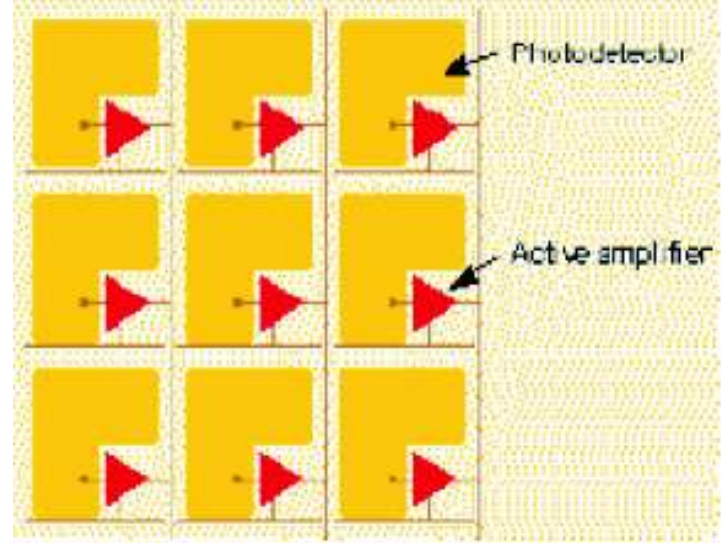

Fig. 6. ACMOS based IR Sensor

\section{Proximity electronics}

Electronic biasing is needed for proper sensing as well as amplifying the signal of each pixel. Moreover, the image is obtained by scanning all the pixels in some order. The later is facilitated by a set of externally generated clock pulses as shown in Fig. 7.
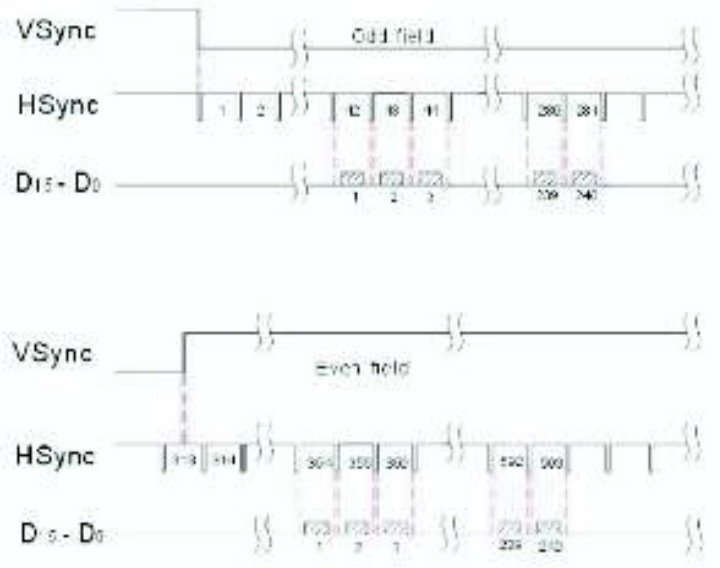

Fig. 7. Timing pulses for scanning [6]

The analog signal from each pixel is digitized using an analog to digital converter (ADC). Besides, it is also possible to set gain and offset of the signals from each pixel. All these are facilitated by the proximity electronics in the camera.

\section{Cryogenics and thermal stabilization}

Most of the photonic IR detectors need to be cooled during operation due to the extreme dark noise they have at room temperatures that will be swamp out the signals. Hence, some sort of cryogenic cooling is mostly necessary for these sensors. Originally Liquid Nitrogen (LNT) in Dewars cooled these detectors. However, this not only 
prevented the cameras being used in any orientation but also there was a need to often refill the LNT while operation for long durations. Nowadays, many IR cameras use close circuit coolers, like Sterling coolers for this purpose. This has greatly increased the ease of operation of these cameras, especially on field.

Besides the above, few IR cameras like the thermal Microbolometer cameras, in spite of the fact they operate at room temperatures needs stabilization of sensor pixel temperature in order the gains and offsets to be stable. Hence, in these cameras thermoelectric stabilizers are used to keep the sensor temperature constant.

\section{Data interface and control}

In many monitoring cameras the two-dimensional digital image array signals so generated is converted in to video signals and displayed on monitors like a TFT LCD colour monitors. Here, false colour display and colour bars are used to differentiate the temperature at different points in the image.

However, for more quantitative work and for permanent storage and manipulation of acquired images they are generally transferred to a PC or a laptop. Moreover, there may be also a need to control the camera during its operation. This may be achieved by varying the set parameters of the camera by the connected external computers.

The two requirements mentioned above are facilitated by proper transfer of data to and fro the computer. For control it is usual to use protocols like RS 232 . Where as the digital image may be transferred using RS 420 or serial buses like USB and Firewire or at times using frame grabbers.

\section{Image processing and control software}

The digital image so transferred to the computers are not only stored and displayed, they can also be manipulated to extract important information. All these are performed by the Image Processing Softwares. These are nowadays available either commercially (eg. MATLAB) or can be developed in house.

As mentioned above, the cameras can also be controlled from the computer. That will need specific drivers and control software that is camera dependent.

\section{INFRARED MICROBOLOMETER CAMERAS}

Uncooled thermal cameras, like Microbolometer detector based cameras, have several advantages over cooled photonic cameras. They are compact, lightweight and inexpensive. They are also more reliable since they do not need cryogenic coolers. Besides, they do not need much maintenance too. Other advantages are low power consumption and large spectral response. Even though they have lower sensitivities and slower response times, owing to the previously mentioned advantages, the usage of these cameras, both in civilian and military applications, have increased tremendously over the last few years.

Microbolometers work on the principle of detection of variation of resistance of certain material due to the temperature change by absorption of IR radiation. Hence, material with high Temperature Coefficient of Resistance (TCR) is preferred. The main material that are used as IR sensors are amorphous silicon, vanadium oxide etc. This paper will deal mainly with a-silicon based Microbolometers. An important aspect is that the heatsensing portion of the detector should be insulated from the heat sink in order to improve its sensitivity. This is usually achhieved by a bridge mechanism. One can also notice the reflector at the bottom to reflect the IR light and increase the sensitivity. The Fig. 8 shows the structure of a single pixel of the micro bolometer.

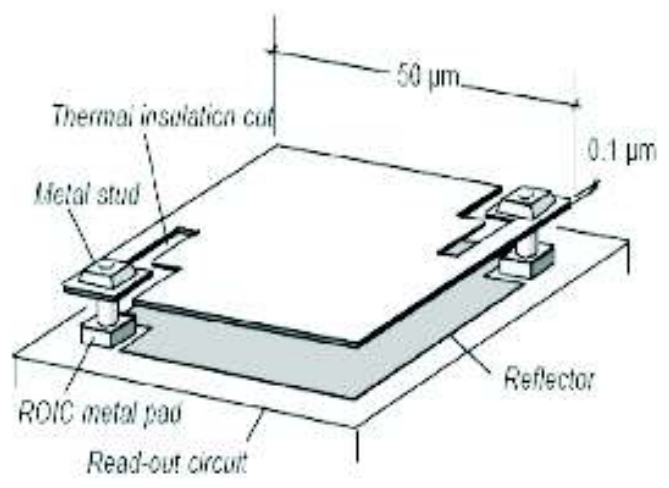

Fig.8. Structure of a single microbolometer pixel [5]

The gap between the reflector and the sensor allows peak sensitivity at $10 \mathrm{~m}$. Fig. 9 . shows SEM picture of a Microbolometer array.

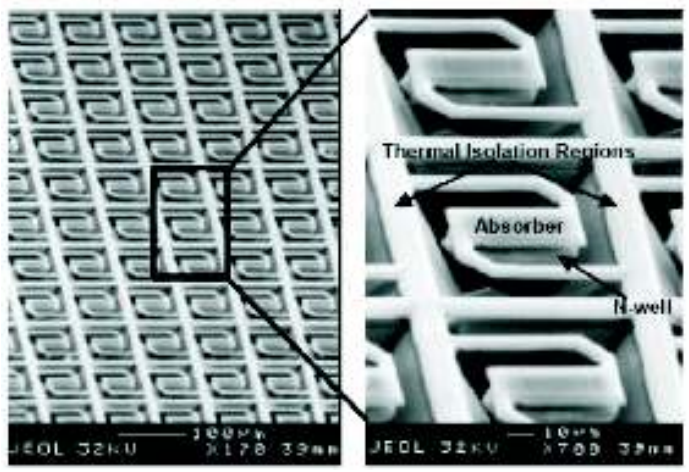

Fig. 9. SEM picture of a Microbolometer FPA [3] 
The whole array is fabricated over a CMOS Read Out Integrated Circuit (ROIC). The array is encapsulated and evacuated to reduce the heat flow from the pixel sensor elements.

Shows SEM picture of a Microbolometer array. The whole array is fabricated over a CMOS Read Out Integrated Circuit (ROIC). The array is encapsulated and evacuated to reduce the heat flow from the pixel sensor elements.

Microbolometer arrays are sensitive to $813 \mathrm{~m}$ range of IR radiation. They have a noise level of (NETD) of 100 $\mathrm{m}$ oK. They can be operated till $60 \mathrm{~Hz}$. The sensors generally use a thermoelectric cooler for stabilization.

The technology involved in fabrication is that of MEMS. They are compatible with the CMOS ROIC fabrication technology that is used in memory chip manufacturing for microprocessors.

\section{AVAILABLE TECHNOLOGIES IN INDIA}

We see that in order to manufacture Uncooled Microbolometer cameras following technologies are required.

1. IR optics design

2. Sensor fabrication

3. Electronics design

4. DataAcquisition and Transfer skills

5. Image Processor and Control software development

6. Cryogenics

Experiences in optics design using ray-tracing codes are available in many research institutions, universities and even in some commercial enterprises in India. Lens fabrication facilities are also available to some extent with in the country. Electronics design and fabrication expertise needed for this type of work is abundantly available in many places here. Owing to the great strides taken by the IT field in India during the last one decade, development of Image processing and Control software may not pose a great challenge. So is the experience with data acquisition and transfer. In the same way, many national research institutions and some IITs have adequate know how in the field of Cryogenics.

However, baring few defense laboratories, design and fabrication experience in the MEMS and Sensor and Arrays is lacking. There may also be a problem in finding foundries for making these sensor ICs also. This is one of the important challenges that face the infrared imaging community in India.

\section{CONCLUSION}

From the above we can see that indigenous manufacturing of IR cameras, that too Uncooled Microbolometer cameras, have great application potential and economic benefit for India. Most of the skills required for are available, though in a scattered and isolated way, in many institutions and Industries with in India itself. However, there is an urgent need to for developing experience in design and manufacturing of infrared sensor arrays with in India. Hence, owing to the tremendous advantage the indigenous manufacturing of Uncooled Bolometer Cameras posses, there is a need for a concentrated development programme. In order to achieve this goal in an economical way and in the shortest possible time, it would be advantageous to synergise all the locally available knowledge and skills by a joint national project. Hence, there is a strong case for a multi institutional project to develop Uncooled Microbolometers with in India.

\section{REFERENCES}

[1] For basic of thermal imaging visit http://www.temperatures.com/thermalimaging.html

[2] Xavier P.V. Maldague, 2001, Theory and Practice of Infrared Technology for Nondestructive Testing, John Wiley \& Sons, Inc. New York.

[3] Deniz Sabuncuoglu Tezcan, Selim Eminoglu, Orhan Sevket Akar, and Tayfun Akin, January 2001, The $14^{\text {th }}$ IEEE Int. MicroElectroMechanical Systems Conf. (MEMS 2001), Interlaken, Switzerland, pp. 566-569

[4] D.S. Tezcan, F. Koçer, and T. Akin, June 1999, The $10^{\text {th }}$ Int. Conf. on Solid-State Sensors\&Actuators (TRANSDUCERS'99), Sendai, Japan, pp.610-613

[5] J.L. Tissot, J.L. Martin, E. Mottin, M. Vilain, J.J. Yon, J.P. Chatard, 2000, Infrared technology and Applications XXVI, Vol.4130, pp. 473-479.

[6] GUIDIR, 2005, IR108B Infrared Imaging Module User Manual, Publication No: IR108B UM 006, Wuhan Guide Infrared Technology Co., Ltd., 